\title{
Near-field imaging of optical diffraction radiation generated by a 7-GeV electron beam
}

\author{
A. H. Lumpkin, W. J. Berg, N. S. Sereno, D. W. Rule, ${ }^{*}$ and C.-Y. Yao \\ Advanced Photon Source, Argonne National Laboratory, Argonne, Illinois 60439, USA
}

(Received 4 May 2005; published 26 February 2007)

\begin{abstract}
We report the first unambiguous demonstration of near-field imaging of optical diffraction radiation (ODR). The source of the ODR was an aluminum metal reflective surface with a 7-GeV electron beam passing nearby its single edge. Because of the high Lorentz factor $\gamma$ involved, appreciable ODR is emitted at visible wavelengths even for impact parameters of 1 to $2 \mathrm{~mm}$, so standard imaging techniques were employed. The experimental results are compared to a simple near-field model. We show that the ODR signals are sensitive to both beam size and position. Applications to multi-GeV beams in transport lines in the major synchrotron radiation facilities, x-ray free-electron lasers, energy recovering linacs, and the International Linear Collider are possible.
\end{abstract}

DOI: 10.1103/PhysRevSTAB.10.022802

PACS numbers: 41.60.Cr, 41.60.Ap

\section{INTRODUCTION}

There is a long-standing interest in the ability to monitor a high-energy and/or high-power electron beam's transverse size in a nonintercepting (NI) manner [1]. Although this is routinely done in ring accelerators by imaging using the NI properties of optical and x-ray synchrotron radiation (OSR and XSR), this option is not available in linear accelerators or linear transport lines. This need has become critical in an accelerator complex such as the Advanced Photon Source (APS) where top-up operations involve injection of 7-GeV beam through a transport line into the storage ring every two minutes [2]. Beam position is routinely measured using $\mathrm{rf}$ beam position monitors (BPMs), but NI beam-size measurements have been a challenge. One candidate for assessing the transverse beam size is optical diffraction radiation (ODR), which is emitted when a charged-particle beam passes near the edge of a conducting surface or through an aperture in the surface. This basic radiation mechanism has been investigated for years [3-5], but to date most applications involving beam-size analysis have been based on detection of small changes in the far-field angular distribution due to beam-size effects [6-12]. These effects are often partially obscured by beam-offset and beam-divergence contributions. More recently, an unusually low-divergence beam $(\sim 1.5 \mu \mathrm{rad})$ allowed ODR far-field data from a slit aperture to be analyzed for beam-size effects [13]. These data were obtained via a scanning-mirror plus detector geometry, but the scan took ten minutes. A preliminary theoretical discussion of the use of near-field ODR was given in Ref. [9].

In this paper, we report for the first time the observation of ODR by imaging with focus at the object (the metal screen) on which it is produced, i.e., the near-field source image. These near-field observations lead to an alternate paradigm for using ODR to measure relative beam size

\footnotetext{
*Permanent address: Carderock Division, NSWC, West Bethesda, MD 20817, USA.
}

using direct near-field optical imaging to assess the beamsize dimension parallel to a metal plane's edge. For example in this case, the relative horizontal size and position can be measured from a vertically inserted mirror edge on a single beam pulse (see the inset of Fig. 1).

We show that the ODR peak signal intensity dependence is consistent with $\exp (-2 d / \gamma \lambda)$, where $d$ is the distance from beam center to the screen edge, $\gamma$ is the Lorentz factor, and $\lambda=\lambda / 2 \pi$ is the reduced observation wavelength, and that the ODR image vertical profile is qualitatively consistent with our simple analytical model. Basically, this is revealed through the 2D image/map of the induced current distribution on the screen. That is, we detect the spatial distribution of fields due to the passing charge as revealed through the photons emitted by the metal screen. Our observations are consistent with the idea that the material's electrons are accelerated and thus radiate as the particle beam passes below the metal screen's edge with an impact parameter $d$ of a few $\mathrm{mm}$. We show that this "image" or electromagnetic "reflection" is representative of the beam's horizontal size and position and can therefore be used as an NI relative monitor of those parameters. In the limit as the impact parameter goes through zero and the beam is completely on the screen, the radiation reduces to the more well-known optical transition radiation (OTR), which is emitted as a chargedparticle beam transits the vacuum-metal interface. The backward OTR and ODR are both proportional to the Fresnel reflection coefficients squared. By using the OTR image of the beam's current distribution and rf BPM readings as references, we validated these NI diagnostic techniques based on ODR.

\section{EXPERIMENTAL AND ANALYTICAL BACKGROUND}

\section{A. Experimental background}

The ODR experiment was performed at the APS facility, which includes an injector complex with an rf thermionic 


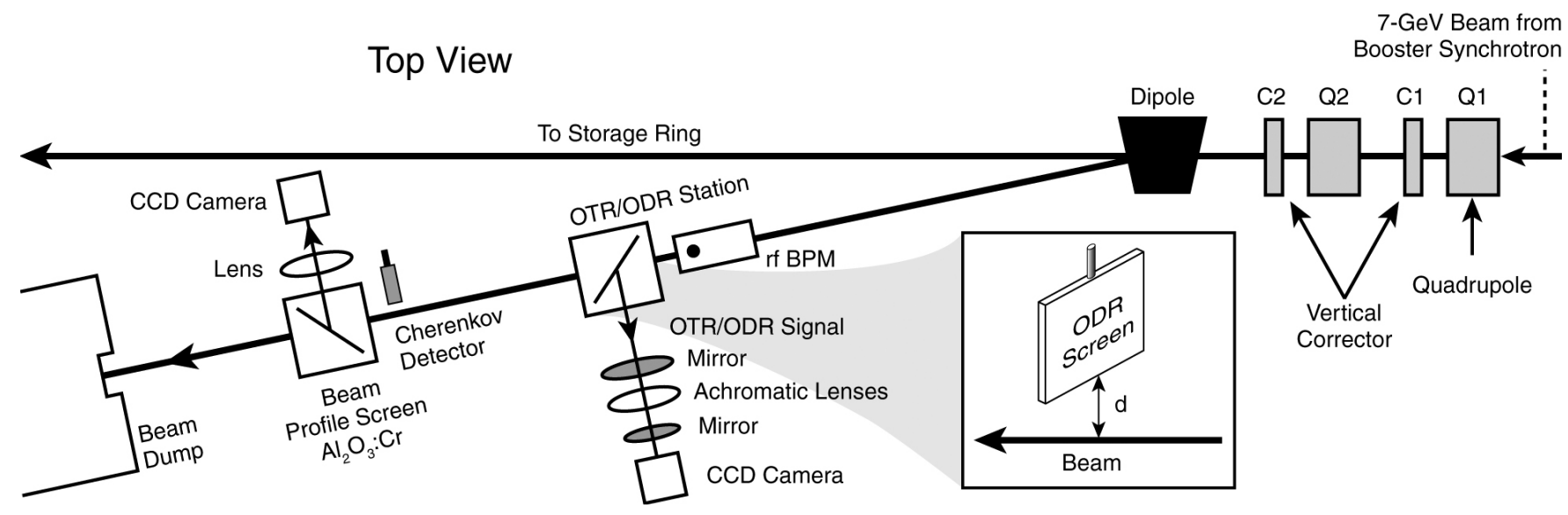

FIG. 1. Schematic of the BTX line at the APS with the rf BPM, OTR/ODR station, Cherenkov detector, beam profiling screen, and beam dump. The upstream dipole whose center is $5.84 \mathrm{~m}$ from the station and the vertical correctors are used to direct the beam to the station.

gun, an S-band linear accelerator, a particle accumulator ring (PAR) that damps the linac beam at $325 \mathrm{MeV}$, a booster synchrotron that ramps the energy from 0.325 to $7 \mathrm{GeV}$ in $220 \mathrm{~ms}$, and the $7-\mathrm{GeV}$ storage ring. In a $24-$ singlets fill in the storage ring, we need to inject 2.5 to $3.0 \mathrm{nC}$ per shot into the ring at two-minute intervals to maintain the stored beam current within $1 \%$ of $100 \mathrm{~mA}$. The $\mathrm{rf}$ beam position monitors provide position readings in an NI manner, but the beam size and quality are only monitored with OSR in the injector rings. The booster synchrotron to storage ring transport line has no NI monitor of beam size at present. We can check beam size with intercepting screens, but the goal is to employ NI techniques during top-up operations. At the exit of the booster synchrotron, a dipole allows the beam to be directed to an alternate line (BTX) that ends with a beam dump. The center of this 1.94-m-long magnet is $5.84 \mathrm{~m}$ upstream of the diagnostics station. At the magnet exit, the vacuum chamber has a $25-\mathrm{mm}$-diameter aperture that transitions to the 50 -mm-diameter beam pipe. This spur line has been used to develop our ODR diagnostics. The setup includes the upstream corrector magnets, quadrupoles, and this dipole, and then an rf BPM (single-plane striplines only), the OTR/ODR imaging station, a localized beam-loss monitor based on a Cherenkov radiation detector, the Chromox beam profiling screen, and the beam dump, as schematically shown in Fig. 1. The stripline BPM was oriented for vertical position only for the initial series of experiments in testing the ODR phenomenon, and then it was rotated 90 degrees to provide direct, complementary horizontal position readings to the ODR centroids in the subsequent set of experiments.

The ODR converter is a polished $\mathrm{Al}$ blade/mirror that is $1.5 \mathrm{~mm}$ thick, $30 \mathrm{~mm}$ wide, $30 \mathrm{~mm}$ tall, and is mounted with its surface normal at $45^{\circ}$ to the beam direction on a stepper assembly (see the Fig. 1 inset). The assembly provides vertical positioning with an overall accuracy of $\pm 10 \mu \mathrm{m}$ over a span of $27.5 \mathrm{~mm}$. Because of radiation safety issues, the blade was constrained to move a maximum of $7.5 \mathrm{~mm}$ below the centerline. The OTR and ODR signals were directed by turning mirrors and relay optics to a Sony Model XC CCD camera located $1.8 \mathrm{~m}$ from the source. The initial relay optics consisted of five, $50-\mathrm{mm}$ diameter achromats which included one triplet [with focal lengths (fl) of $150 \mathrm{~mm}, 250 \mathrm{~mm}$, and $100 \mathrm{~mm}$ ] at $z=$ $340 \mathrm{~mm}$; one singlet (fl of $250 \mathrm{~mm}$ ) at $z=690 \mathrm{~mm}$; and one singlet $(\mathrm{fl}=250 \mathrm{~mm})$ at $z=1540 \mathrm{~mm}$. The camera sensor was at $z=1790 \mathrm{~mm}$. The magnification was chosen to cover the blade edge assembly and resulted in calibration factors of $55 \mu \mathrm{m} / \mathrm{pixel}$ in $x$ and $45 \mu \mathrm{m} / \mathrm{pixel}$ in $y$. These were validated by the physical size of the screen image and by stepping the blade edge known amounts. Beam charge at the station could be varied from 0.4 to $3.5 \mathrm{nC}$ as extracted from the booster synchrotron at $7 \mathrm{GeV}$. The OTR signal almost saturated the camera for a beam charge of $0.4 \mathrm{nC}$.

\section{B. Potential background light sources}

In order to assess any background contributions that might contaminate the ODR signal, we calculated the OSR distribution from the main dipole that is $5.84 \mathrm{~m}$ upstream of the station. For the field of $0.9 \mathrm{~T}$ and a bending radius of $25 \mathrm{~m}$, the OSR at $500 \mathrm{~nm}$ is a very low intensity, flat (to 20\%) background on the outboard side above and below the midplane as seen in Fig. 2. The OSR polarization component parallel to the bend plane is 4 times stronger than the perpendicular component. In fact, we calculate the total OSR intensity is 100 times weaker than the predicted ODR at $d=1.0 \mathrm{~mm}$ as shown in Fig. 3. A simple polarization experiment confirmed that the ODR image has a stronger vertical polarization component than horizontal, which thus precludes the dipole OSR as the source. We actually detected some OSR from other upstream sources 


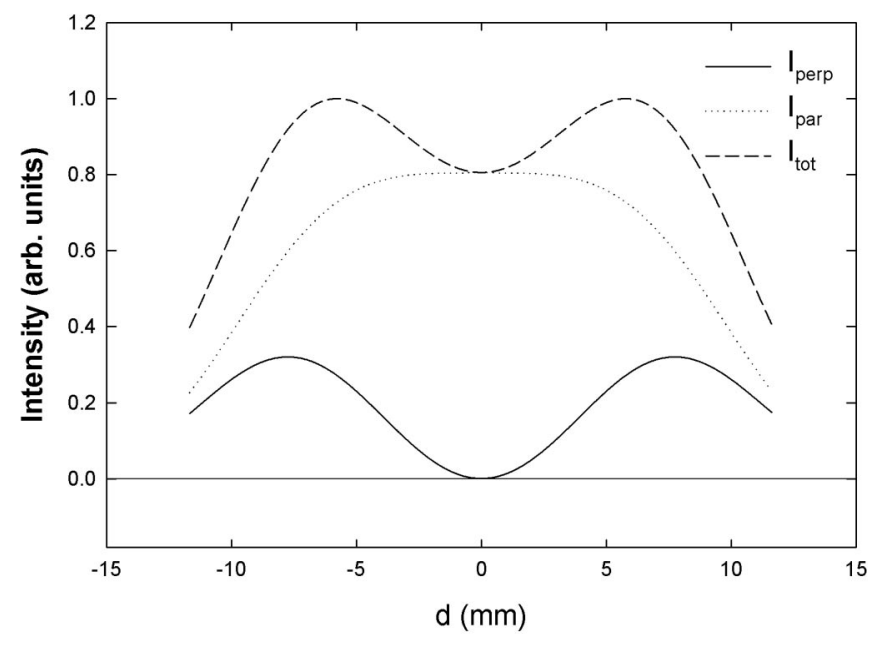

FIG. 2. A calculation of the angular distribution of the out-ofplane OSR from the upstream dipole projected at a distance of $5.8 \mathrm{~m}$ and using a wavelength of $500 \mathrm{~nm}$.

such as the correctors or quadrupoles, so we adjusted the upstream vertical correctors until that background light was at or below the beam centerline. This reduced the background for ODR analysis above the beam centerline. The authors of Refs. [12,13] note a $20 \%$ background effect in their far-field data that they could not subtract.

Another possible source of light contamination of the observed ODR could be OTR from a beam halo intercepting the screen. In order to rule this out, a Cherenkov detector [14,15] operating as a beam-loss monitor was used in some of our studies to verify that the beam halo at $3.5 \mathrm{nC}$ was negligible above the normal Gaussian profile intensity at impact parameters of 4 to $5 \sigma_{y}$, where $\sigma_{y}$ is the rms vertical size. Experiments were performed both by taking five images for each blade position with an MV200 videodigitizer, which provided online analysis, and by tracking 15 to 20 measurements [EPICS process

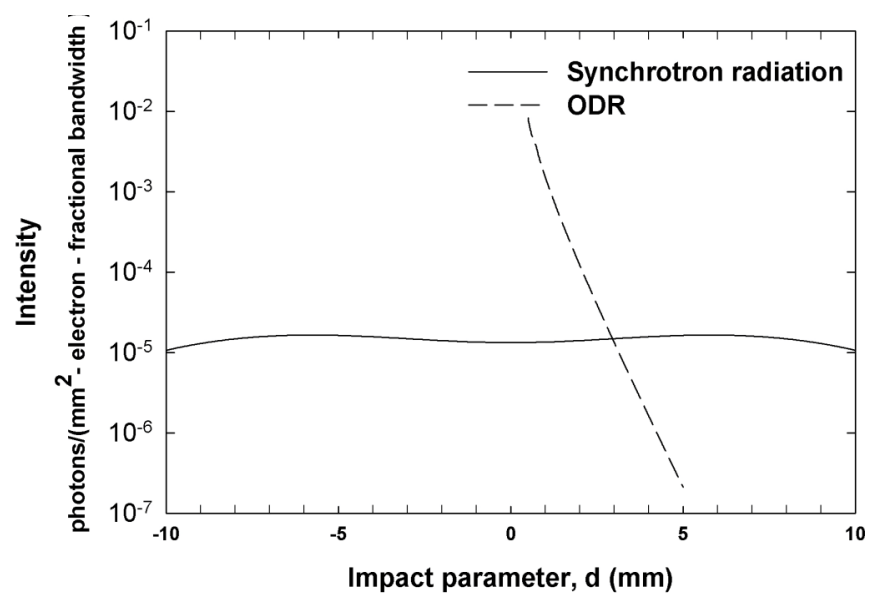

FIG. 3. A comparison of the calculated projected OSR from the upstream dipole with a beam bending radius of $25 \mathrm{~m}$ and the ODR intensities at the OTR/ODR screen. variables, or PVs, correlated using the self-describing data set (SDDS) toolkit [16]] as the script commanded the blade edge position. The rf BPM readings, an upstream current monitor, the Cherenkov detector readings, and several parameters from the image processing ( $x$-centroid fit, $x$-sigma fit, $y$-centroid fit, $y$-sigma fit) were tracked as well as analysis based on peak intensity to full-width half-maximum intensities and peak positions. The fits to beam profiles were Gaussian distributions since the beam had been damped by the synchrotron radiation mechanism in the booster synchrotron prior to extraction to the beam line. The Cherenkov detector readings indicated there was very little beam halo above the Gaussian profile at distances beyond $4-5 \sigma_{y}$.

\section{Analytical model considerations}

Some further background discussion on ODR is warranted. As stated before, ODR is produced when an electron beam passes near a region where different dielectric materials are present. This is generally a vacuum-to-metal interface, and the theory [5] is usually for the far-field, Fraunhofer diffraction pattern produced by a beam passing through apertures or slits in conducting planes. In the present case, our optical system effectively integrates over angle and frequency since it is focused on the ODR source itself, i.e., the near-field image on the screen. Therefore we propose a simplified model of the near field based on the method of virtual quanta described by Jackson [17] in dealing with the photonlike fields of relativistic beams. We convolve the electron beam's Gaussian distribution of sizes $\sigma_{x}$ and $\sigma_{y}$ with the field expected from a single electron at point $P$ in the metal plane. We wish to calculate the incoherent sum of radiation from all beam particles in a pulse emitted from a given point on the ODR radiator, i.e., at $\boldsymbol{u}=\boldsymbol{P}-\boldsymbol{r}_{o}$, where $\boldsymbol{P}$ is the field point with respect to the origin and $\boldsymbol{r}_{o}$ is the position of the beam centroid with respect to the origin. The impact parameter is $\boldsymbol{b}=\boldsymbol{u}-\boldsymbol{r}$, where $\boldsymbol{r}=\boldsymbol{r}(x, y)$ denotes a position in the beam measured from the beam centroid. We then can write the differential spectral intensity as

$$
\begin{aligned}
\frac{d I}{d \omega}(\mathbf{u}, \omega)= & \frac{1}{\pi^{2}} \frac{q^{2}}{c}\left(\frac{c}{v}\right)^{2} \alpha^{2} N \frac{1}{\sqrt{2 \pi \sigma_{x}^{2}}} \frac{1}{\sqrt{2 \pi \sigma_{y}^{2}}} \\
& \times \iint d x d y K_{1}^{2}(\alpha b) e^{-\left[x^{2} /\left(2 \sigma_{x}^{2}\right)\right]} e^{-\left[y^{2} /\left(2 \sigma_{y}^{2}\right)\right]},
\end{aligned}
$$

where $\omega=$ radiation frequency, $v=$ particle velocity $\approx$ $c=$ speed of light, $\quad q=$ electron charge, $\quad N \quad$ is the particle number, $\alpha=1 / \gamma \lambda$, and $K_{1}(\alpha b)=$ $K_{1}\left[\alpha \sqrt{\left(u_{x}-x\right)^{2}+\left(u_{y}-y\right)^{2}}\right]$ is a modified Bessel function. If we measure the unpolarized light intensity $I$, this should be proportional to $\left|E_{x}\right|^{2}+\left|E_{y}\right|^{2}$, resulting in the $K_{1}^{2}$ 
dependence. The separate horizontal and vertical polarizations can be modeled in a similar manner. The incoherent photon intensity is proportional to $N$, the number of electrons, in contrast to the case of coherent diffraction radiation in the far infrared (FIR) [18], which is enhanced by $N^{2}$.

\section{RESULTS AND DISCUSSIONS}

\section{A. Observations of near-field ODR characteristics}

The crux of the experiment is shown in Fig. 4. First, Fig. 4(a) shows an OTR image of the 7-GeV beam. The observed beam sizes were $\sigma_{x}=1375 \pm 75 \mu \mathrm{m}$ and $\sigma_{y}=$ $200 \pm 25 \mu \mathrm{m}$ for a 0.4-nC beam. In Fig. 4(b), the image ascribed to ODR for an impact parameter of $d=1.25 \mathrm{~mm}$ and $Q=3.3 \mathrm{nC}$ is shown. The light emission starts $1.25 \mathrm{~mm}$ above the beam centerline (the dashed line in the figure). This edge distance is $6 \sigma_{y}$ beyond the vertical beam center, but comparable to $\gamma \lambda$ as discussed below. At this $d$ the ODR dominates by a factor of 60 over the calculated broad, low intensity OSR from the dipole (see Fig. 3), but ODR is about 10 times weaker than OTR in absolute terms. Further evidence for the assignment of the signal to ODR is the tracking of the signal's vertical peak position versus the blade edge position as plotted in Fig. 5. A good linear fit was found for $d=0.5$ to $3 \mathrm{~mm}$ from the beam centerline. The rf BPM readings show the vertical beam position was the same to $\pm 40 \mu \mathrm{m}$ during the scan. Because of the exponential-like decay of ODR peaks vertically, the signal profiles are strongest at the smaller impact parameters in contrast to what would be expected from a broad, nearly constant OSR background. In 5-frame sum image data we actually see ODR peak signal out to the $3250-\mu \mathrm{m}$ point, more than twice the nominal distance scale $d \cong \gamma \lambda$ as seen in Fig. 6 . These data are characterized by an exponential scale factor of $\gamma \lambda / 2=909 \mu \mathrm{m}$ with an effective wavelength of $834 \mathrm{~nm}$. This was done with beam charges of $3.3 \mathrm{nC}$, a factor of only eight more than used in the OTR imaging. In addition, the ODR $y$ profile is shown for an impact parameter of $500 \mu \mathrm{m}$. There
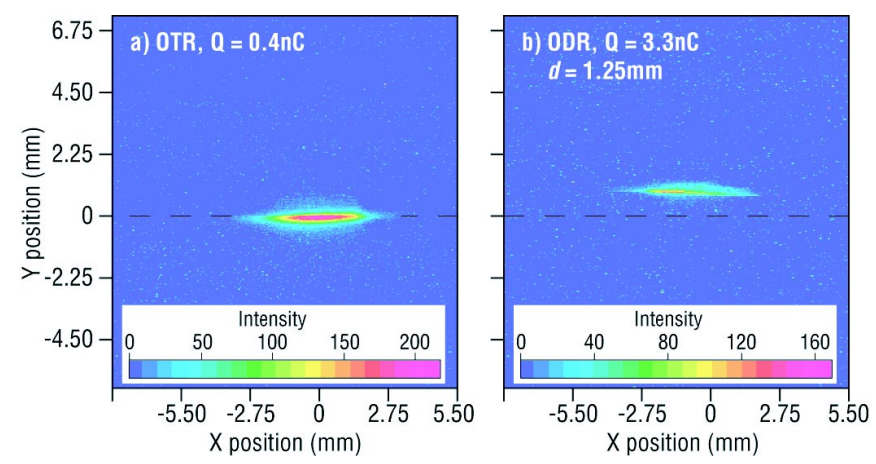

FIG. 4. (Color) Images produced by the 7-GeV beam: (a) OTR with $Q=0.4 \mathrm{nC}$ and (b) ODR with $d=1.25 \mathrm{~mm}$ and $Q=$ $3.3 \mathrm{nC}$. The dashed line is the beam centerline.

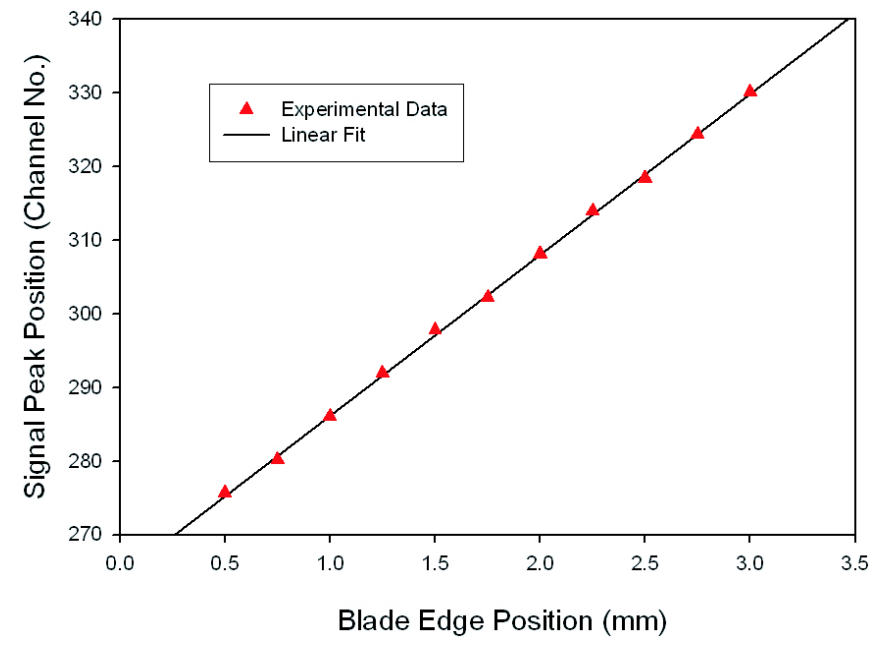

FIG. 5. (Color) Plot of the ODR signal peak vertical position versus the distance from the beam to blade edge. Since the beam vertical position did not change on this scale as monitored by the rf BPM, the ODR signal peak is coming from near the metal screen edge.

is a truncation of this profile at around $1800 \mu \mathrm{m}$ compared to the peak intensity data which we attribute to vignetting caused by a variation in the effective angular aperture across the vertical field of view. Figures 4-6, represent our original set of near-field ODR studies that did not have the benefit of laser prealignment of the optical system.

Our subsequent series of experiments were performed with an altered optical system that allowed a far-field imaging option and included laser prealignment. We also exchanged our camera for one with enhanced infrared sensitivity, the Sony XC-EI30 model. Figure 7 shows a

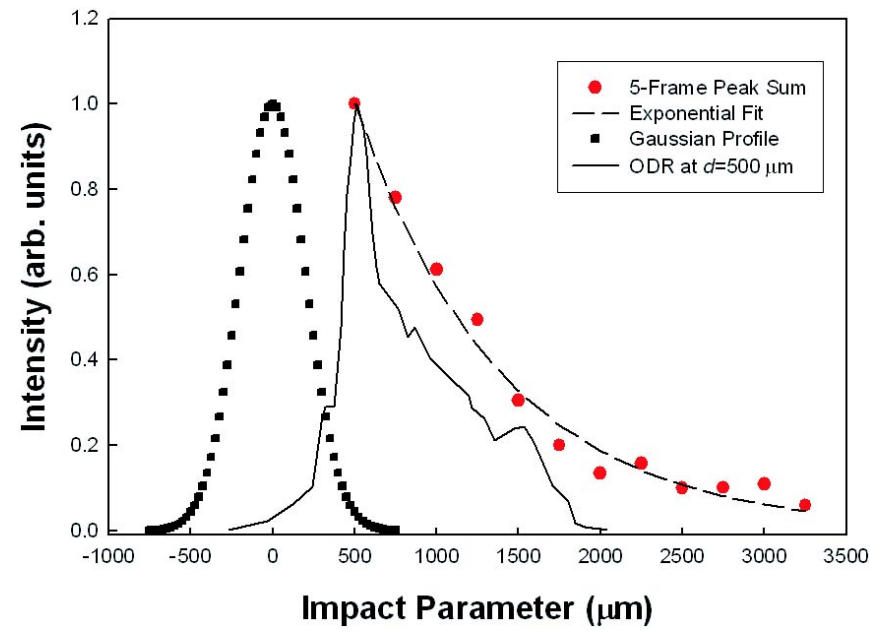

FIG. 6. (Color) Plots of the ODR peak intensity versus the distance from the beam to blade edge or impact parameter, an exponential curve fit to the data with $\gamma \lambda / 2$ equal to $909 \mu \mathrm{m}$, and an initial ODR $y$ profile are shown. A Gaussian fit to the OTR beam distribution for $\sigma_{y}=200 \mu \mathrm{m}$ centered at $d=0$ is also shown for reference. 


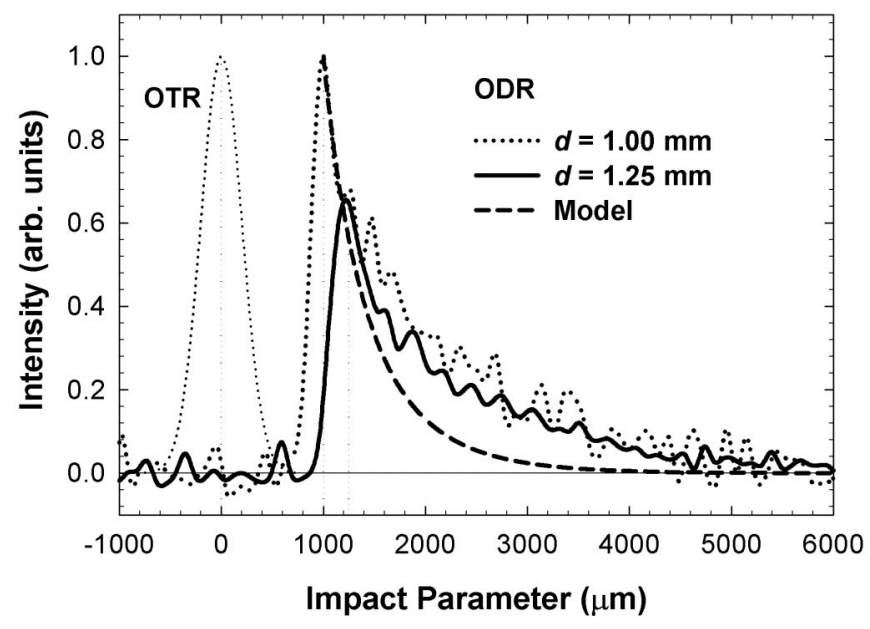

FIG. 7. Comparison plots of a Gaussian fit to the OTR beam distribution for $\sigma_{y}=200 \mu \mathrm{m}$ centered at $d=0$, the $d=1000$ (dotted line) and $1250 \mu \mathrm{m}$ (solid line) ODR image vertical profiles, and the Eq. (1) model result (dashed line) scaled to the vertical profile data.

simple interpretation of these later experiments. We compare the rapid decrease of the Gaussian fit of the OTR vertical beam profile with $\sigma_{y}=200 \mu \mathrm{m}$ to the ODR profile data, which no longer exhibit the sharp truncation as seen in Fig. 6. In addition, we show that the $d=1000$ - and 1250- $\mu \mathrm{m}$ ODR image vertical profiles overlap at distances larger than $1250 \mu \mathrm{m}$. There have been separate normalizations for the OTR and ODR data to a value of 1.0 in Fig. 7. Our model Eq. (1) results qualitatively reproduce the shape of the vertical profiles after normalizing to the experimental value at $d=1000 \mu \mathrm{m}$. No bandpass filters were used in the initial experiments so our model was integrated over all visible wavelengths, weighted by the camera response function. Clearly, we see ODR signal far beyond the OTR image profile, and the model supports this effect. There is an uncertainty in the absolute starting position of the two vertical profiles of approximately $\pm 100 \mu \mathrm{m}$ since they were acquired on different dates and the rf BPM had been rotated for horizontal plane coverage only. Given this uncertainty, the model agrees reasonably well with the data out to $\sim 1500 \mu \mathrm{m}$. The data fall off more slowly than the model results at larger impact parameters as if a longer effective wavelength is involved. Additional experiments employing bandpass filters and polarizers may probe the extent of the details described by this simple model. These are still the first direct comparisons of any model to near-field ODR data anywhere.

Additionally, we tested the horizontal position sensitivity of ODR signal by steering the beam horizontally (i.e., parallel to the blade edge) using the upstream dipole while the blade edge was positioned $0.75 \mathrm{~mm}$ above the beam centerline. This same dipole had been used in the beamloss commissioning tests when the beam position was tracked with OTR. The SDDS experiment tracked the

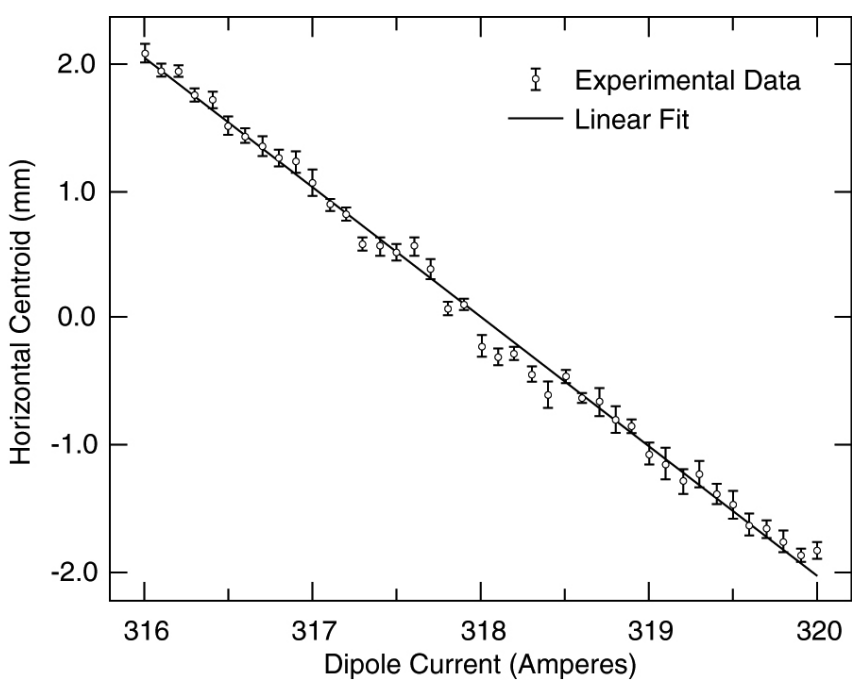

FIG. 8. Plot of the horizontal ODR image position (centroid) versus the upstream dipole magnet current supply value $(A)$. The impact parameter is $0.75 \mathrm{~mm}$ for this case, and the Cherenkov detector indicated negligible beam losses.

processed video image PVs including the ODR image centroids, the Cherenkov detector readings, and the $\mathrm{rf}$ BPM readings. The plot in Fig. 8 shows the ODR image centroid position versus the dipole current settings. The error bars are the standard deviation based on 10-shot sampling. Position changes along the $x$ axis of less than 50 to $100 \mu \mathrm{m}$ can be sensed, even though the beam size is nominally $\sigma_{x}=1375 \mu \mathrm{m}$. The vertical rf BPM confirmed that the vertical beam positions varied by no more than $10 \mu \mathrm{m}$ during the dipole current scan. The variation of position with power supply current, or $\mathrm{mm} / \mathrm{A}$ sensitivity, agrees with that of the OTR data. Therefore, the light being imaged is produced locally by the beam in the vicinity of the screen surface and cannot be from upstream OSR effects. Subsequent experiments with the rf BPM stripline rotated 90 degrees for horizontal position tracking, gave $\mathrm{mm} / \mathrm{A}$ sensitivities within $5 \%$ of the ODR results as described in a later section.

\section{B. Nonintercepting beam size and position monitoring applications}

The potential to monitor relative beam size has been discussed in our earlier papers [19-21]. However, in this case we now have the results of vertically polarized data, and the screen, optical mirrors, lenses, and camera were prealigned with a laser positioned on the beam centerline. First, benchmark OTR image profiles were obtained by inserting the $\mathrm{Al}$ metal screen into the beam during a scan of the upstream AQ2 quadrupole field. This quadrupole strongly affects the horizontal beam size at our OTR/ ODR station. Unfortunately, this is at a dispersive point in the lattice so we could not get emittance data cleanly from the quadrupole field scan. However, we do obtain a 


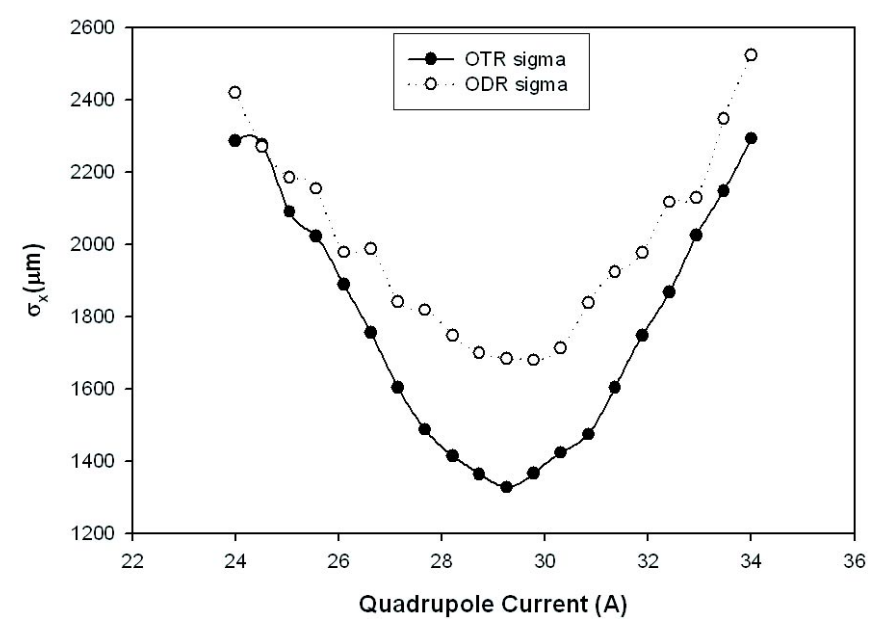

FIG. 9. A direct comparison of the OTR (closed circles) and ODR (open circles) Gaussian-fit horizontal profile sizes during the quadrupole field scan. The vertically polarized ODR tracks the beam-size changes.

test of ODR as a relative beam-size monitor. As seen in Fig. 9, the OTR-measured horizontal beam size varies from $2300 \mu \mathrm{m}$ down to about $1300 \mu \mathrm{m}$. Results of the Gaussian fitting algorithms are shown since the beam shape is essentially Gaussian when extracted from the 7-GeV booster synchrotron. We also show the observed vertically polarized ODR image profiles with an impact parameter of $1.25 \mathrm{~mm}$ during a similar quadrupole field scan. The vertically polarized ODR horizontal image profiles track the beam-size changes as seen qualitatively by the shape of the curve with AQ2 current. This direct comparison of the OTR and ODR is seen in the Fig. 9 combined plot of their fitted rms values. The ODR profiles are about 5\% to $25 \%$ larger than the corresponding OTR profiles from the largest beam size to the smallest, respectively. The ratio of ODR/OTR for the size scan is plotted in Fig. 10, which

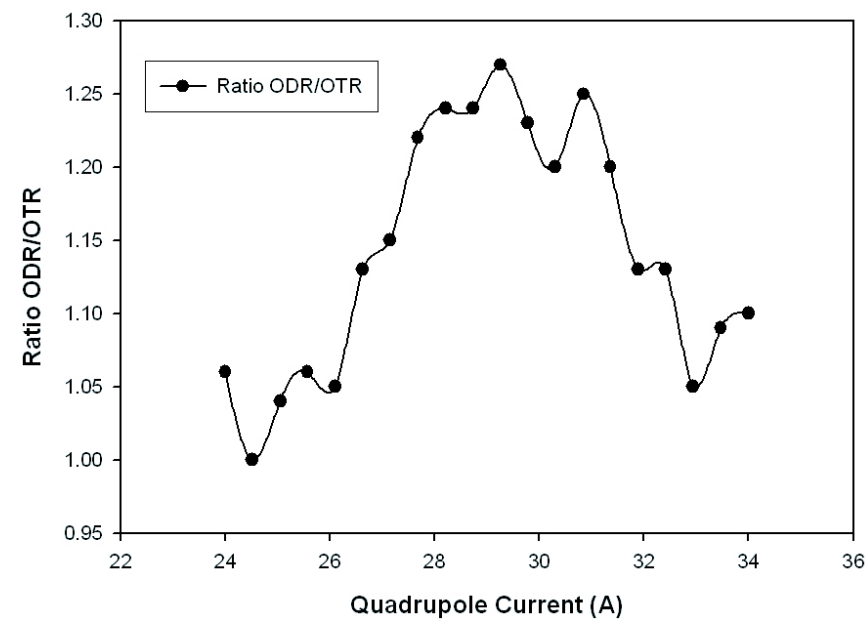

FIG. 10. The ratio of ODR/OTR horizontal image sizes for different beam sizes during the AQ2 quadrupole scan. This ratio is noticeably smaller than for unpolarized data reported earlier.

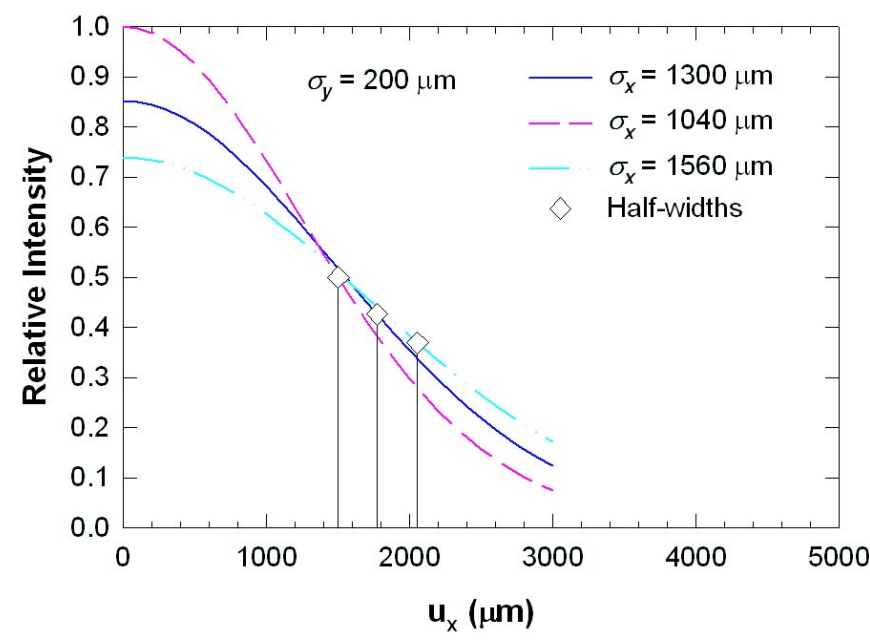

FIG. 11. (Color) Analytical model results for the effects on the unpolarized ODR horizontal profiles for a variation of the beam size by $\pm 20 \%$ around the $1300-\mu \mathrm{m}$ value with $d=1000 \mu \mathrm{m}$.

could act as a look-up table for the beam-size monitor. As can be seen, at the minimum size of $1300 \mu \mathrm{m}$ for the OTR, the ODR fit value is only $25 \%$ larger. This is much better than our factor of 2 results with unpolarized ODR observed on the same shift for this minimum beam focus as previously reported [20]. The vertical polarization component would appear to be more reliable and direct in monitoring the actual beam size to better than $10 \%$, once you have the ratio table or plot for these conditions.

In order to assess further the sensitivity of ODR for potential beam diagnostic applications, we have numerically evaluated Eq. (1) for fixed $\gamma \lambda$ and found qualitative support for the observed ODR Gaussian shape along the $x$ axis, the dependence on $x$-beam size, and the exponential-like decay of the peak intensity along the $y$ axis. Our calculations revealed easily detectable changes of the ODR image size by $\sim \pm 15 \%$ with beam-size changes of $\pm 20 \%$ at a central value of $\sigma_{x}=1300 \mu \mathrm{m}$ as shown in Fig. 11. In this case of a highly elliptical beam, we calculated very little change in the ODR $y$-profile for $20 \%$ changes in the $\sigma_{y}=200 \mu \mathrm{m}$ value. We also calculated that for smaller beam sizes we should use a correspondingly smaller impact parameter. For example, we found ODR image $x$-size changes of $25 \%$ correlate to a beam-size change from $\sigma_{x}=20$ to $50 \mu \mathrm{m}$ with a fixed $y$-impact parameter of $100 \mu \mathrm{m}=5 \sigma_{y}$, while keeping a constant $\sigma_{y}=20 \mu \mathrm{m}$. The clear effects at the halfmaximum level of the ODR profile are shown in Fig. 12. This regime of beam sizes is expected in x-ray freeelectron lasers (XFELs), energy recovering linacs (ERLs), APS Upgrade, CEBAF, and after the damping ring of the proposed International Linear Collider (ILC). Because of the high average power of the beams, the NI aspect is critical.

In addition, we revisited our relative beam position measurements first done with unpolarized ODR and com- 


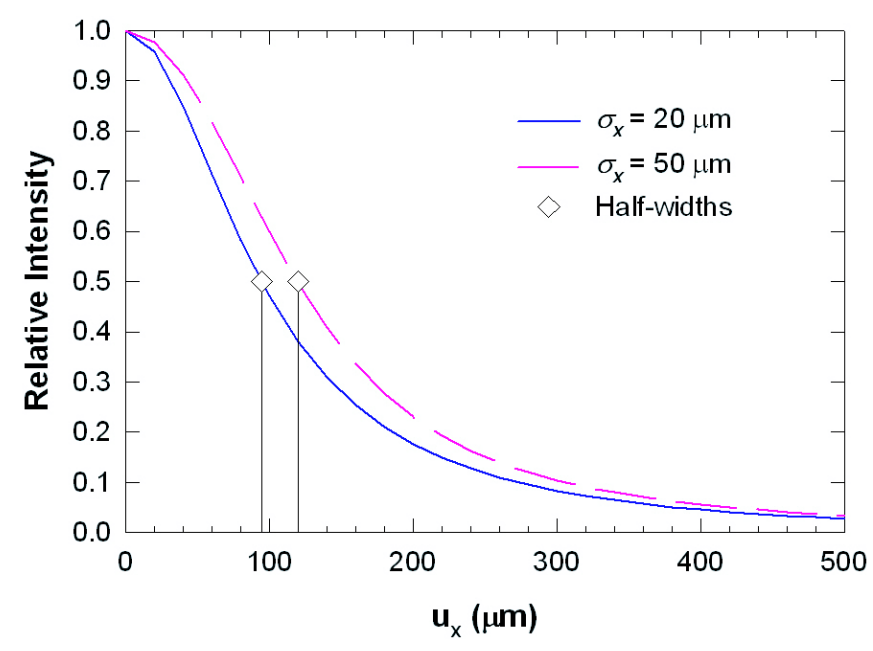

FIG. 12. (Color) Analytical results for the change in unpolarized, horizontal ODR profiles for a change in beam size from 20 to $50 \mu \mathrm{m}$ while holding the $y$ value constant at $20 \mu \mathrm{m}$. The impact parameter is $100 \mu \mathrm{m}$ for the $7-\mathrm{GeV}$ beam. This is relevant to XFEL beam-size monitoring and other advanced accelerators.

pared to the rf BPM and OTR values [20]. In this case, we again used vertically polarized ODR with an impact parameter $d=1.25 \mathrm{~mm}$ and a vertical size $\sigma_{y}$ of $200 \mu \mathrm{m}$ or less. The plot in Fig. 13 actually compares the image centroid values from both OTR and ODR to the horizontal BPM readings. The OTR and ODR data overlap each other almost completely. This was done with the beam size $\sigma_{x}=$ $1300 \mu \mathrm{m}$ with the AQ2 quadrupole field set for the beamsize minimum. Again we believe the vertically polarized ODR component benefits the measurement sensitivity in the horizontal axis. Sensitivity at the 50- to $100-\mu \mathrm{m}$ rela-

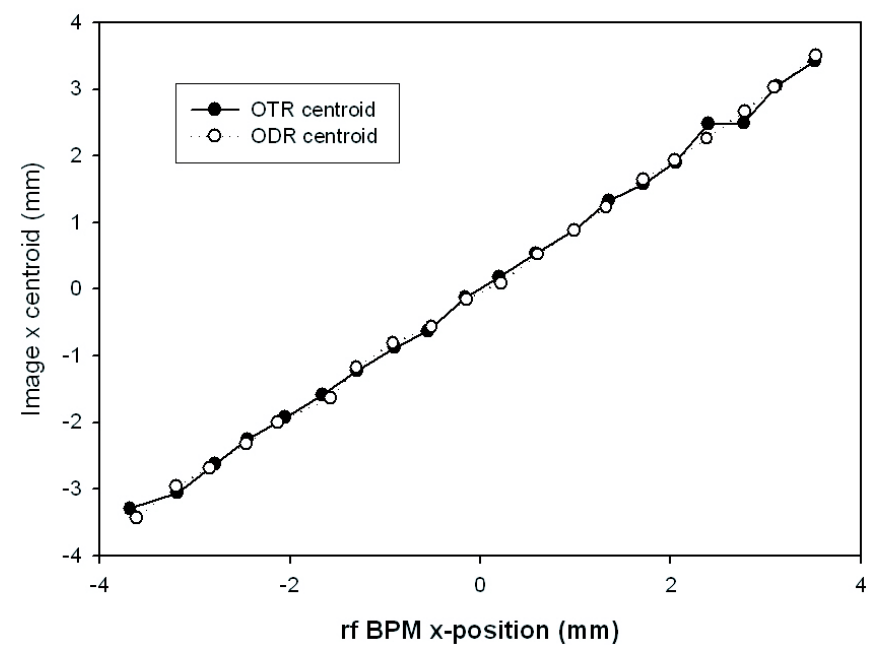

FIG. 13. A plot of the OTR and ODR centroid value changes versus the nearby $\mathrm{rf}$ BPM values in mm during a scan of the upstream dipole current supply (and magnet fields). Horizontal position information can be reliably obtained from the vertically polarized ODR image centroids. tive position level is attained. As in the case of beam-size monitoring, for the much smaller beam and impact parameter in the XFEL case, we would expect much better position sensitivity (sub-10 $\mu \mathrm{m}$ ) subject to signal level. Correspondingly, a vertical single edge of a metal screen or aperture can be employed to obtain information on vertical position and beam size using the orthogonal or horizontal polarization component.

\section{SUMMARY}

In summary, we report the first near-field experimental imaging study of ODR from a single-edge metal surface. We have demonstrated that ODR techniques are clear candidates for tracking relative changes in beam size (related to emittance and quality) and relative position in the transport line between the synchrotron and storage ring during our top-up mode. In principle, a second orthogonal blade could be used to determine beam size and position in the vertical plane (a rounder beam would help), and in general, slits or other apertures should be useful for other specific beam implementations. In applications with damped beams from a booster synchrotron, the expected Gaussian beam profile makes modeling simpler. This technique works with relatively large beam divergences, in our case 30 times that of Ref. [13]. Near-field ODR imaging should also have potential application to other thirdgeneration rings that use top-up, as well as to the highpower systems such as the accelerator drivers for XFELs, light sources based on ERLs, and that proposed for the ILC. Effective relative size sensitivity at the $20-$ to $50-\mu \mathrm{m}$ regime for a Gaussian beam is indicated for an impact parameter of $5 \sigma_{y}=100 \mu \mathrm{m}$ according to our model, while $10-\mu \mathrm{m}$ sensitivity for position should be achievable. These initial results have helped to elucidate the ODR mechanism, and it is hoped that they will also stimulate further development of nonintercepting diagnostics for present and future accelerator-based facilities.

\section{ACKNOWLEDGMENTS}

The authors acknowledge B. Yang for input on optics issues; B. Rusthoven and A. Barcikowski for the mechanical design of the stepper motor and ODR blade assembly; A. Pietryla and C. Gold for the Cherenkov detector installation; L. Emery for beam loss calculations; and J. Xu and S. Shoaf for controls for the ODR station and Cherenkov detector. We acknowledge O. Singh and W. Ruzicka for project support. One of the authors (A.H.L.) acknowledges past and recent discussions on OTR/ODR with R. Fiorito (University of Maryland) and H. Loos (SLAC), respectively. This work was supported by the U.S. Department of Energy, Office of Basic Energy Sciences, under Contract No. W-31-109-ENG-38. 
[1] Proceedings of the ICFA 17th Advanced Beam Dynamics Workshop on Future Light Sources, Argonne National Laboratory, Argonne, IL, 1999 (Argonne National Laboratory, Argonne, IL, 2000).

[2] A. H. Lumpkin, W. J. Berg, and B.X. Yang, AIP Conf. Proc. 648, 433 (2002).

[3] F. G. Bass and V. M. Yakovenko, Sov. Phys. Usp. 8, 420 (1965).

[4] A. P. Kazantsev and G. I. Surdutovich, Sov. Phys. Dokl. 7, 990 (1963).

[5] M.L. Ter-Mikaelian, High Energy Electromagnetic Processes in Condensed Media (Wiley/Interscience, New York, 1972).

[6] M. Castellano, Nucl. Instrum. Methods Phys. Res., Sect. A 394, 275 (1997).

[7] Y. Shibata et al., Phys. Rev. E 52, 6787 (1995).

[8] A. P. Potylitsyn, Nucl. Instrum. Methods Phys. Res., Sect. B 145, 169 (1998).

[9] R. B. Fiorito, D. W. Rule, and W. D. Kimura, AIP Conf. Proc. 472, 725 (1999); W. D. Kumura, R. B. Fiorito, and D. W. Rule, Proceedings of the 1999 Particle Accelerator
Conference, 1999, p. 487.

[10] D. W. Rule, R. B. Fiorito, and W. D. Kimura, AIP Conf. Proc. 390, 510 (1997).

[11] R. B. Fiorito and D. W. Rule, Nucl. Instrum. Methods Phys. Res., Sect. B 173, 67 (2001).

[12] T. Muto et al., Phys. Rev. Lett. 90, 104801 (2003).

[13] P. Karataev et al., Phys. Rev. Lett. 93, 244802 (2004).

[14] Alan S. Fisher, AIP Conf. Proc. 451, 95 (1998).

[15] A. Pietryla, W. Berg, and R. Merl, 2001 Particle Accelerator Conference, Chicago, IL, 2001, p. 1622.

[16] H. Shang, Proceedings of PAC2003, 2003, p. 3470.

[17] J. D. Jackson, Classical Electrodynamics (John Wiley and Sons, New York, 1975), Sec. 15.4.

[18] A. H. Lumpkin, N.S. Sereno, and D. W. Rule, Nucl. Instrum. Methods Phys. Res., Sect. A 475, 470 (2001).

[19] Alex H. Lumpkin, Nucl. Instrum. Methods Phys. Res., Sect. A 557, 318 (2006).

[20] A. H. Lumpkin et al., AIP Conf. Proc. 868, 384 (2006).

[21] A.H. Lumpkin et al., Proceedings of FEL06, Berlin, Germany, 2006, p. 710. 